\title{
El proceso de investigación. De la idea disparadora al artículo científico
}

\author{
Di Masso, R.J.1,3; Gayol, M. del C.'; Tarrés, M.C. ${ }^{2,3}$ \\ ${ }^{1}$ Cát. Genética, Fac. Cs. Veterinarias, Universidad Nacional de Rosario (UNR), Ovidio Lagos y Ruta 33, \\ 2170 Casilda, Argentina. Tel/Fax. 03464423377. ${ }^{2}$ Cát. Biología, Fac. Cs. Médicas, UNR. ${ }^{3}$ CIC-UNR. \\ E-mail: rjdimasso@gmail.com
}

\begin{abstract}
Resumen
Di Masso, R.J.; Gayol, M. del C.; Tarrés, M.C.: El proceso de investigación. De la idea disparadora al artículo científico. Rev. vet. 25: 2, 146-153, 2014. Los momentos o etapas reconocibles de manera genérica en el proceso de investigación científica, los pasos del método hipotético-deductivo de aplicación corriente en las ciencias fácticas naturales y la clásica estructura IMRYD del artículo de investigación científica presentan una lógica común. Esta comunicación busca exponer dicha lógica transparentando los vínculos entre ellos y remarcando la doble condición -actividad y producto- de la ciencia.
\end{abstract}

Palabras clave: momentos del proceso de investigación, método hipotético-deductivo, estructura IMRYD.

\begin{abstract}
Di Masso, R.J.; Gayol, M. del C.; Tarrés, M.C.: The research process. From the trigger idea to the scientific article. Rev. vet. 25: 2, 146-153, 2014. The so-called moments or generically recognizable phases in the research process, the steps of the hypothetical-deductive method commonly applied in the factual sciences and the classical IMRAD structure of scientific research articles share a common logic. This paper seeks to expose this logic, making evident the links between them and emphasizing the dual role of science both as an activity as well as a product.
\end{abstract}

Key words: steps of the research process, hypothetical-deductive method, IMRAD structure.

La ciencia comienza hoy a desvelar sus verdaderos rostros...., no es ni diosa ni ídolo, tiende a confundirse cada vez más con la aventura humana de la que ha surgido.

Edgard Morin

\section{A modo de introducción}

En ocasiones la universidad suele ser caracterizada como un ámbito de transmisión de conocimiento. Si se considera la distinción efectuada por algunos autores ${ }^{9}$ y se acepta que la información es un conjunto de datos puestos en contexto mientras que el conocimiento es el producto de la incorporación de esa información a la trama propia de cada sujeto cognoscente, lo correcto sería referirse a la universidad como un ámbito de transmisión de información. Pero restringir la función de la universidad a la mera reproducción de saberes ya disponibles es despojarla de lo que debe ser una de sus características más conspicuas: la producción de conocimiento crítico, entendiendo por tal ese saber cuya

Recibido: 5 marzo 2014 / Aceptado: 30 mayo 2014 adquisición está lejos de ser espontánea, un saber metódico, sistemático que fundamenta sus afirmaciones, que pretende la objetividad y que es generado mediante estrategias legitimadas por la comunidad científica ${ }^{5}$.

El objetivo de este texto es presentar, en forma resumida, el camino a recorrer desde que, en el marco del denominado contexto de descubrimiento, se gesta la idea que dispara el proceso de investigación, hasta que el producto del mismo se socializa y se pone a disposición de la sociedad a la que genuinamente pertenece, exponiendo la lógica de base común que comparten los momentos del proceso, los pasos del método hipotético-deductivo de aplicación generalizada en las ciencias fácticas naturales y la estructura del artículo de investigación científica.

\section{Los momentos del proceso de investigación}

La ciencia es a la vez producto y actividad. El producto es un tipo particular de saberes a los que, por su origen, se adjetiva como científicos. La actividad es la investigación, que para diferenciarla de otras modalidades, o para asignarle más especificidad al término, 
también se la adjetiva como científica. Estos dos aspectos: investigación científica y conocimiento científico, son componentes identificables en la mayoría de las definiciones de ciencia aportadas por diversos autores. Para algunos, por ejemplo, la ciencia como producto es un sistema de ideas establecido provisionalmente ${ }^{2}$. La noción de sistema es central en tanto indica que esas ideas que la humanidad ha generado acerca del comportamiento de parcelas determinadas de la realidad, no se acumulan caóticamente sino que conforman una red intrincada a la que se suman permanentemente nuevos aportes que la amplían y la modifican.

Pensar la ciencia como un sistema de ideas nos permite comprender también que ella no es la realidad misma sino representaciones mentales que la humanidad ha construido a lo largo de su historia, por lo que se dice que es un producto de la cultura. La ciencia como actividad supone un proceso que, si bien con los imprevistos propios de toda actividad humana, está pautado por un orden lógico que permite reconocer etapas o momentos que la caracterizan. El proceso de investigación debe pensarse como un continuo y en él resulta dificultoso reconocer un inicio y un final ciertos. Es como cuando una persona sube a un micro en una estación intermedia y desciende antes del destino final: hubo un trayecto previo del que se puede tener referencias pero del que no se ha sido partícipe necesario y habrá un recorrido posterior que le es posible imaginar pero que tampoco le tendrá necesariamente como protagonista. Habitualmente, entonces, se acostumbra restringir el proceso al período de tiempo que transcurre desde el momento que toma forma la idea disparadora hasta que se escribe el trabajo.

Es en ese trayecto, de duración variable y con sus idas y vueltas, que se suceden conjuntos de acciones que diferentes autores han agrupado utilizando distintos criterios ${ }^{6,12,13}$. No hay acuerdo entre dichos autores acerca de cómo denominar las fases, etapas o momentos que pueden identificarse en el devenir del proceso asociado a la ciencia como actividad. Independientemente de la denominación utilizada, y de la evidente artificialidad que encierra fragmentar en etapas discretas un proceso que es en esencia continuo, desde un punto de vista didáctico es útil adoptar algunos de los criterios disponibles. Uno de tales criterios, por ejemplo, reconoce cuatro momentos a los que denomina lógico, metodológico, técnico y teórico o de síntesis ${ }^{11}$.

Otros agregan a esta secuencia un quinto momento: el momento comunicativo ${ }^{10}$. En el momento lógico el científico, trabajando dentro de los límites de un marco teórico dado, realiza observaciones, se formula preguntas que representan los problemas de conocimiento a resolver, indaga en lo que ya se sabe sobre esa cuestión problemática que llama su atención, recorta la realidad con un objetivo determinado y propone, a modo de hipótesis, una respuesta tentativa al interrogante inicial. En el momento metodológico, plantea una estrategia particular para enfrentar sus ideas acerca de los hechos con los hechos mismos por medio de la experiencia. En el momento técnico lleva efectivamente a la práctica la estrategia planeada en el momento metodológico lo que le permite recolectar información y procesarla para contrastar empíricamente la hipótesis. En una de sus etapas finales el proceso vuelve sobre sí mismo en el denominado momento teórico o de síntesis retornando así a la conjetura inicial y tomando una decisión acerca de su corroboración o su refutación parcial o total.

Este esquema secuencial momento lógico-momento metodológico-momento técnico-momento teórico, no es un círculo que se cierra sobre sí, sino una espiral que al intentar regresar a la pregunta que le dio origen produce un nuevo comienzo a partir del surgimiento de nuevos interrogantes. Pero el proceso no finaliza con la síntesis teórica porque queda aún un paso: poner ese nuevo saber a disposición de la comunidad científica para dar la oportunidad a que nuevas contrastaciones empíricas independientes lo confirmen o no y le permitan integrarse al corpus disciplinar -conjunto de conceptos y teorías propios de esa rama particular de la ciencia- en constante crecimiento y rediscusión. Este nuevo momento hace a una de las características esenciales del conocimiento científico, la de ser comunicable ${ }^{2}$ y recibe el nombre de momento comunicativo. El artículo de investigación científica es una de las estrategias de socialización del conocimiento crítico $\mathrm{y}$, como tal, su publicación forma parte del momento comunicativo. La Figura 1 resume esquemáticamente la sucesión de momentos mencionados y la Figura 2 incluye dicha sucesión en una secuencia temporal que reconoce un "antes" y un "después" que acompaña al "durante" descrito.

\section{Un modelo del proceso de investigación}

Tomando como base los momentos descriptos, se intentará delimitar las acciones que habitualmente se realizan en todo proceso de investigación, para lo cual se ha efectuado la adaptación de un esquema ${ }^{12}$ como modelo del proceso de investigación. Como se mencionó previamente, diferentes autores han presen-

\section{Momentos del proceso de investigación}

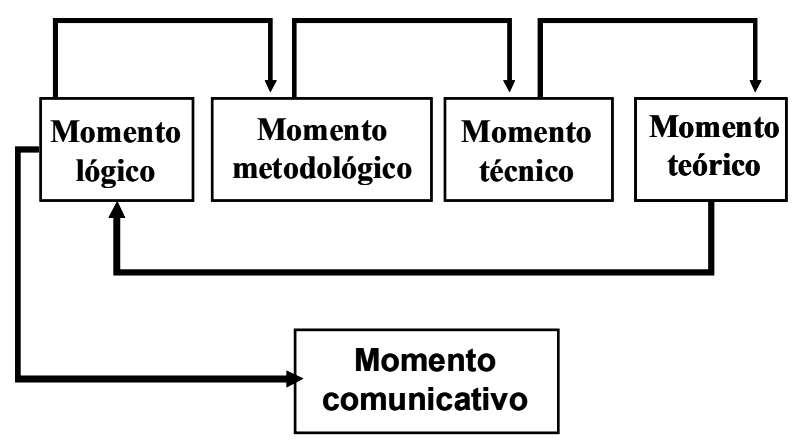

Figura 1. Representación esquemática de las acciones que en un orden lógico se suceden en el marco del proceso de una investigación. 


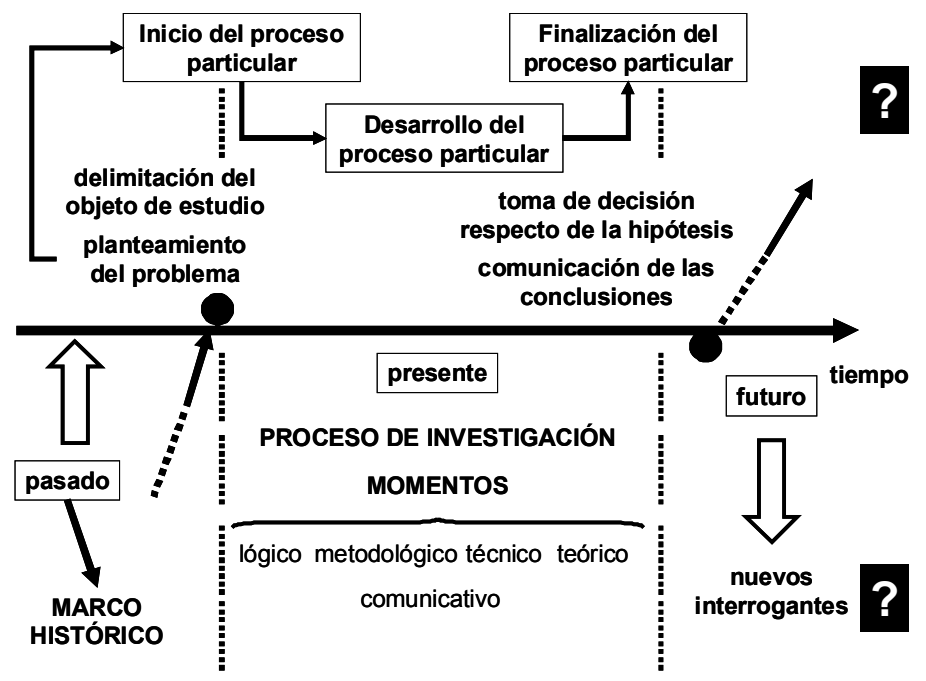

Figura 2. El desarrollo del proceso de investigación en la secuencia temporal del antes, el durante y el después.

tado diversos esquemas de pasos sucesivos que intentan describir las etapas del proceso, pero el esquema presentado en la Figura 3 se distingue por una característica específica: no es lineal. Con ello se pretende poner en relieve el carácter dinámico de la actividad de investigación, de modo que no se conciba el proceso como teniendo un principio y un fin definitivos, sino más bien como un trabajo continuo, donde cada investigación particular es parte de un esfuerzo mucho mayor en el desarrollo de los conocimientos científicos. Por otro lado se observará que el modelo plantea etapas paralelas en su desenvolvimiento. Ello tiene por objeto mostrar que no hay verdaderamente un orden único en el trabajo sino que existen tareas que se desarrollan de un modo simultáneo, que se complementan y determinan mutuamente.

El primer elemento, la definición de un área temática, implica la selección de un campo del saber que tiene unidad interna pero que abarca una problemática mucho más reducida que las disciplinas, y aún que las especialidades, en las que suele ubicarse. El área temática es un referente simbólico y, en tal sentido es un enunciado sobre un conjunto de fenómenos reales. Una vez elegido el tema de interés se construye el objeto de estudio como fenómeno real al que remite el tema. Esta última consideración remarca una cuestión no siempre reconocida: el tema de investigación se elige pero el objeto de investigación se construye.

El tema elegido podría ser, por ejemplo, "la seguridad laboral en el medio rural" y, sobre esa base, construir como objeto concreto de estudio en un momento particular "el uso de los elementos de protección personal por parte de los trabajadores rurales que aplican plaguicidas en la zona tal". Esta diferencia entre tema y objeto de investigación se vincula con la habitual distinción entre línea de investigación y programa de investigación. Los proyectos que forman parte de una línea de investigación comparten un determinado tema, mientras que aquellos que conforman un determinado programa de investigación comparten el objeto de estudio.

La segunda etapa consiste en la formulación o el planteamiento del problema, señalando que un problema de conocimiento se presenta cuando se logra determinar qué es lo que el investigador no sabe dentro de un área temática determinada, cuando se establece una especie de frontera entre lo conocido y lo no conocido y se toma la decisión de indagar sobre esto último. A este respecto, se ha presentado una distinción interesante entre tres tipos de ignorancia ${ }^{3}$. Existe una ignorancia trivial que no produce mayormente inquietud y que se relaciona con eso que suele llamarse cultura general. Ignorar quién escribió una obra musical o un libro o quién recibió el último premio Nobel de la paz serían ejemplos aplicables a esta categoría. Otras dos modalidades cuyo denominador común es que producen inquietud en el que ignora, son la ignorancia teórica y la ignorancia erudita. En ambos casos hay desconocimiento de algo.

La diferencia entre estas dos categorías estriba en que en la primera de ellas el conocimiento del que se carece está disponible y, por lo tanto, dicha ignorancia puede superarse estudiando. Por el contrario, en la segunda, el conocimiento no está disponible y sólo se puede superar investigando. El problema de investigación

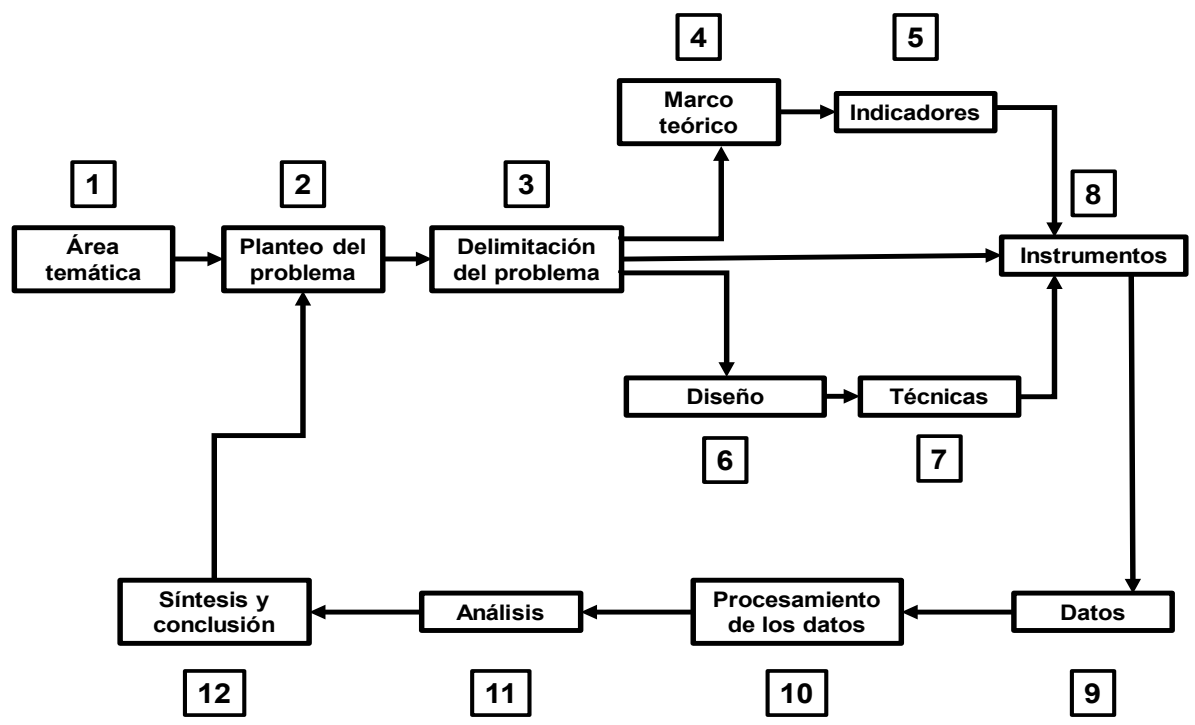

Figura 3. Modelo gráfico del proceso de una investigación. Tomado de Sabino C.A.: El proceso de investigación, $3^{\circ}$ ed., Lumen, Bs.As. p. 46. 
es, entonces, una pregunta que formulada en el marco de un caso de ignorancia erudita. También recibe el apelativo de ignorancia específica, afirmándose que "un requisito previo de la investigación en cualquier campo es admitir que se ignora algo que merece conocerse" 4 .

La delimitación del problema (etapa 3) incluye la tarea de fijar los objetivos del trabajo a desarrollar, tanto generales como específicos. Pero para llegar al enunciado de los objetivos se deben cumplimentar una serie de pasos que algunos resumen en los siguientes cinco: delimitación del problema en el tiempo, delimitación en el espacio, delimitación semántica o de significado de los términos utilizados, enunciado de oraciones tópicas o propósitos iniciales de tipo general $\mathrm{y}$, por último, delimitación de los recursos disponibles para llevar a cabo la empresa ${ }^{6}$.

La elaboración del marco teórico (etapa 4) está estrechamente ligada al planteamiento del problema y, en los hechos, ambas cuestiones se van desenvolviendo casi simultáneamente, ya que no puede plantearse un problema de investigación delimitando lo que no se sabe sobre algo si no se revisa y asimila previamente lo que ya se conoce acerca ello. El marco teórico excede, en realidad, el tema de los antecedentes específicos del problema a resolver. La idea disparadora del proceso de investigación surge de la teoría. Pero ¿de qué teoría? ¿Desde qué lugar "miran" la realidad los sujetos cognoscentes? Resulta evidente que la pregunta que se formula sobre un problema de investigación, un problema biológico, por ejemplo, puede variar según se lo haga en el marco de la biología molecular o en el marco de la biología poblacional.

Se trata de dos niveles diferentes de organización de la materia viva, con sus particularidades. Del mismo modo un problema en el ámbito de la producción animal se enfocará de diferente manera si se lo piensa en un sistema intensivo que prioriza el rendimiento individual a si se lo sitúa en un sistema extensivo que enfatiza la productividad colectiva. El marco teórico nos dice entonces desde qué lugar se va a "mirar" el problema. Se plantea que el marco teórico permite establecer en qué tradiciones se apoya y a qué tradiciones enfrenta un investigador al decidir poner en marcha un proceso de investigación científica ${ }^{13}$. Estas primeras cuatro fases corresponden al momento lógico.

A partir de la etapa 3 , se produce una bifurcación que implica la realización de trabajos simultáneos o paralelos. Esto quiere decir que todo problema de investigación se presenta bajo una doble faz: por un lado, como un conjunto de conceptos y de proposiciones relativos a esos hechos o fenómenos -línea superior de la Figura 3- y por otro -línea inferior de la misma figura- como una colección de hechos, de fenómenos empíricos, rescatando la circunstancia que la investigación debe atender a la doble naturaleza de lo que se construye como objeto, dado que éste no sólo es un segmento de la realidad, sino que está precisamente constituido como resultado de una labor de conceptualización y de elaboración teórica.
El paso 5 es la continuación operativa del marco teórico. La obtención de indicadores, llamada en ocasiones operacionalización, tiene por objeto la búsqueda de elementos concretos, empíricos, que permitan traducir y medir en la práctica los conceptos que se han definido teóricamente. En el lenguaje habitual se suele nombrar como variables a aquello que bien se manipula o bien se mide -en el sentido amplio del verbo mediren el proceso de investigación. Esta denominación es propia de la estadística que clasifica a las variables en cualitativas y cuantitativas, en continuas y discretas, $u$ otras categorías. Sin embargo, desde un punto de vista metodológico, las variables son campos teóricos y la construcción del objeto de estudio incluye la elección de indicadores adecuados para llevar adelante la etapa práctica del proceso.

De ahí la denominación de operacionalización que se otorga al proceso de pasar del plano teórico al plano empírico, de hacer "operativa" la variable. Se puede estar interesados en estudiar el crecimiento de una especie animal de interés productivo pero "el crecimiento" es un campo teórico mientras que la decisión de qué medir -por ejemplo: el peso corporal, la altura a la cruz, la circunferencia corporal, -cuándo medir-al nacimiento, al destete, a la faena, a intervalos regulares durante el ciclo productivo, -y cómo medir- con balanza, con cinta métrica, son decisiones asociadas a la construcción particular del objeto de estudio y forman parte de la operacionalización de la variable crecimiento.

El paso 6 consiste en el diseño concreto y cumple la función de complementarse con el marco teórico: si éste proporciona el marco conceptual y referencial para el problema, el diseño tiene por misión determinar la forma en que la hipótesis asociada al problema habrá de ser puesta a prueba. Debe haber una coherencia lógica entre lo planteado en el marco teórico y la etapa del diseño. Si se adhiere a una lógica de investigación cualitativa basada en un paradigma interpretativo, se utilizará un diseño solidario con ese posicionamiento el que será diferente del que se hubiera pensado desde una lógica cuantitativa y un paradigma explicativo ${ }^{10}$. Los pasos 5 y 6 conforman el momento metodológico.

Las técnicas de recolección de datos (etapa 7) representan la implementación instrumental del diseño escogido y se concretizan, en sintonía con la etapa 5, en la confección de instrumentos para llevar a cabo dicha recolección (cuestionarios, pautas de observación, reglas de medición). Así, ambas líneas, la empírica y la teórica, confluyen en este elemento con el cual se obtendrán los datos, pensados como unidades de información capaces de posibilitar la elaboración de una respuesta para el problema inicial. Pero esas unidades de información que llamamos datos (paso 9) se obtienen en bruto y necesitan, por tanto, de un trabajo de clasificación y ordenación que habrá de hacerse teniendo en cuenta las proposiciones sobre las que se asienta la investigación. El procesamiento de los datos (elemento 10), cierra el momento técnico. 
Finalmente, con estos datos ya procesados, se retomará la labor propiamente teórica para poder obtener de ellos información que permita elaborar una decisión con respecto a la respuesta tentativa -hipótesis- ofrecida desde la teoría al problema planteado. En tal sentido será preciso analizar críticamente la información (paso 11), sistematizarla y sintetizarla para arribar a conclusiones generales de acuerdo a los datos disponibles (etapa 12). Se arriba, así, al momento teórico de la investigación. Con lo expuesto, se cierra parcialmente el proceso de investigación, ya que, generalmente, la respuesta eficaz a un problema implica la aparición de otros nuevos problemas a investigar. El ciclo se reiniciará a partir del segundo elemento, de un modo circular e inacabable, como lo es en verdad la tarea del hombre por resolver los interrogantes del mundo que lo rodea y de su propia práctica.

La Figura 4 pone en paralelo los momentos con el modelo del proceso de investigación.

\section{El método hipotético-deductivo}

Por método se entiende un conjunto de pasos ligados entre sí por un propósito. Cuando el propósito es generar conocimiento científico, ese conjunto de pasos recibe el nombre de método científico. En la actualidad se discute si puede hablarse de "el método", como si el mismo fuese necesariamente uno o si, por el contrario, existen "los métodos". Estas dos posturas, conocidas como monismo y pluralismo metodológico respectivamente ${ }^{7}$, están relacionadas en gran medida con la debatida división de las ciencias en "formales" y "fácticas", estas últimas abarcando las ciencias naturales y las ciencias sociales ${ }^{1,5}$.

Las ciencias fácticas -como, por ejemplo, la biología- son aquellas cuyos objetos de estudio son entes

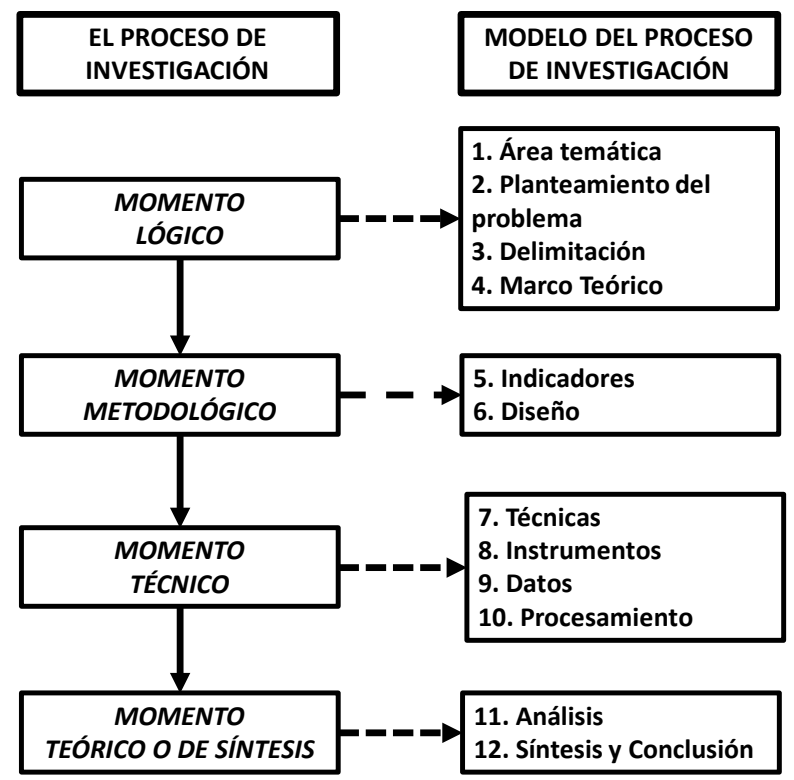

Figura 4. Paralelismo entre los momentos lógicos y las etapas que pueden identificarse en el proceso de investigación. sensibles, hechos con existencia real en el tiempo y en el espacio, a diferencia de lo que ocurre con las disciplinas formales -lógica, matemática- cuyos objetos de estudio -razonamientos, números- son entes ideales, y como tal son intemporales y carecen de existencia espacial. Las ciencias fácticas se subdividen, a su vez, en ciencias naturales y ciencias sociales. Dado que en este caso se alude al ámbito disciplinar de las ciencias fácticas naturales, la aproximación metodológica se restringirá al denominado método hipotético deductivo.

En el marco de la postura hipotético-deductiva, el método científico consiste en:

I. Enfrentar problemas o, lo que es lo mismo, plantear interrogantes conceptuales. Un problema de investigación es una pregunta acerca del comportamiento de un aspecto de la realidad hecha desde un lugar determinado, desde un marco teórico dado. Como se expresó previamente esta particularidad implica que la idea disparadora que da origen al proceso surge de la teoría, que el objeto de estudio no viene dado sino que será construido por el investigador en el transcurso del proceso y que dicha construcción estará mediada por la teoría que la sustenta.

II. Proponer hipótesis, es decir, dar respuestas tentativas a la pregunta del problema. Una hipótesis es una conjetura, una afirmación que se hace respecto del comportamiento de ese aspecto de la realidad que convoca el interés del investigador, cuyo valor de verdad se desconoce pero que inicialmente se presupone cierta. Esa afirmación es tentativa porque en el momento que se la enuncia representa una idea y no se conoce si los hechos la apoyarán o no.

III. Aplicar la lógica para averiguar qué implica esa afirmación, es decir, deducir consecuencias observables. Si la hipótesis es cierta, si lo que el investigador supone es como él lo afirma, entonces deberá ser capaz de encontrar hechos que confirmen sus suposiciones. Esos hechos que busca surgen por deducción. La deducción, es un tipo de inferencia lógica que va del planteo general al hecho particular, o de un planteo con un grado de generalidad mayor a otro que, sin ser necesariamente particular, presenta menor grado de generalidad. Si se dice que "Todos los mamíferos son homeotermos" y que "El perro es un mamífero", entonces, necesariamente se deduce que "El perro es homeotermo". La deducción no agrega ninguna información a lo que se afirma como hecho general. En el método hipotético-deductivo, la hipótesis es la regla general (Todos los mamíferos son homeotermos). Si dicha regla general es, tal como se supone, cierta, entonces un caso particular de esa regla, es decir un mamífero particular como es el perro deberá presentar el rasgo -ser homeotermo- atribuido a todos los de su clase. A ello se denomina deducir consecuencias observables: definir hechos particulares que, de presentarse, confirmarán la idea expresada en la hipótesis. Esta asociación entre la hipótesis como idea y la deducción de observables como hechos potencialmente confirmatorios de la vali- 


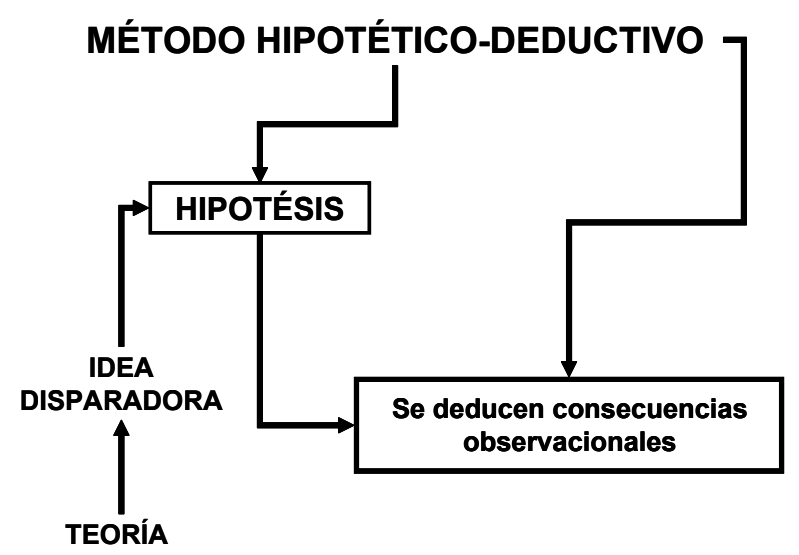

Figura 5. ¿Por qué método hipotético-deductivo?

dez de esa idea, da a esta versión del método científico su denominación de hipotético deductivo (Figura 5).

IV. Confrontar dichas consecuencias con la realidad (contraste empírico).Una vez predicha la consecuencia se debe constatar si ésta efectivamente ocurre en la realidad y no es sólo algo que existe como idea en la mente del investigador. De manera tal que habrá que confirmar que el perro, que sabemos que es un caso de mamífero, presenta efectivamente el rasgo de ser homeotermo que, de acuerdo con la hipótesis, se le ha asignado a todos los de su clase (a todos los mamíferos). Se enfrentará la idea con los hechos y, de acuerdo con el resultado de tal confrontación (a) se abandonará la hipótesis por refutación si resultara ser que el perro, pese a ser un mamífero, no fuese homeotermo y ese único caso es suficiente, entonces, para que no sea posible asignar a todos los mamíferos la condición de homeotermos (un único caso que no presente el rasgo es suficiente para refutar la hipótesis o regla general) o (b) se la conservará total o parcialmente a partir de su corroboración.

Pero evidentemente, el perro no sólo es un mamífero sino que es homeotermo, de manera tal que se corrobora en los hechos aquello que se presupuso como idea. Sin embargo sólo se ha observado un caso (hecho particular) y ello no es suficiente para demostrar la verdad de la regla general. Es por ello que las hipótesis no se verifican, sólo se corroboran. No se puede afirmar la validez del caso general (todos los mamíferos) a partir de un hecho particular (el perro). Esta versión del método hipotéticodeductivo se inscribe en lo que se ha dado en llamar falsacionismo ingenuo en el sentido que da a entender que los científicos abandonan rápidamente sus hipótesis cuando los hechos derivados de la confrontación empírica no acuerdan con sus ideas acerca de esos hechos según han sido planteadas en el modelo teórico postulado. En contraposición con este falsacionismo ingenuo se presenta el denominado falsacionismo sofisticado. En el marco de esta última postura cualquier científico, antes de abandonar su hipótesis, lleva a cabo una serie de acciones confir- matorias de la incapacidad que el modelo teórico planteado tiene para explicar esa parcela de la realidad ${ }^{8}$.

\section{La estructura del artículo de investigación científica}

Se ha afirmado que "el conocimiento cientifico es comunicable, expresable, no es privado sino público. El lenguaje científico comunica información a quienquiera que haya sido adiestrado para entenderlo. La comunicación de los resultados multiplica las posibilidades de su confirmación o refutación. El secreto en materia científica es enemigo del progreso de la ciencia" ${ }^{2}$. Para la UNESCO la finalidad esencial de un artículo científico es comunicar los resultados de investigaciones, ideas y debates de una manera clara, concisa y fidedigna. La Figura 6 resume esquemáticamente las principales características del formato IMRYD (Introducción, Materiales y métodos, Resultados y Discusión) que habitualmente presentan los artículos de investigación científica. El reconocimiento del vínculo lógico existente entre la estructura del artículo científico y el proceso de investigación ha llevado a plantear la lectura crítica del primero como estrategia para el aprendizaje del segundo ${ }^{14}$.

Mucho se ha escrito acerca de esta modalidad de comunicación de los resultados del proceso de investigación científica y cada revista de publicación periódica o journal presenta instrucciones precisas dirigidas a los potenciales interesados en publicar en la misma de modo tal que lo que se presenta a continuación es un sucinto relato sobre la cuestión.

Introducción. Su finalidad es el planteo, claro y simple, del tema de investigación. En este punto pueden reconocerse: (a) la enunciación del problema o interrogante conceptual acompañada en ocasiones con la mención de la idea disparadora del mismo; (b) los antecedentes históricos del problema y (c) el planteo de la hipótesis de trabajo a modo de respuesta tentativa a la pregunta presentada en el problema. En la Introducción del artículo se pone en evidencia el marco teórico desde el que se enfoca y significa el problema y en el que se

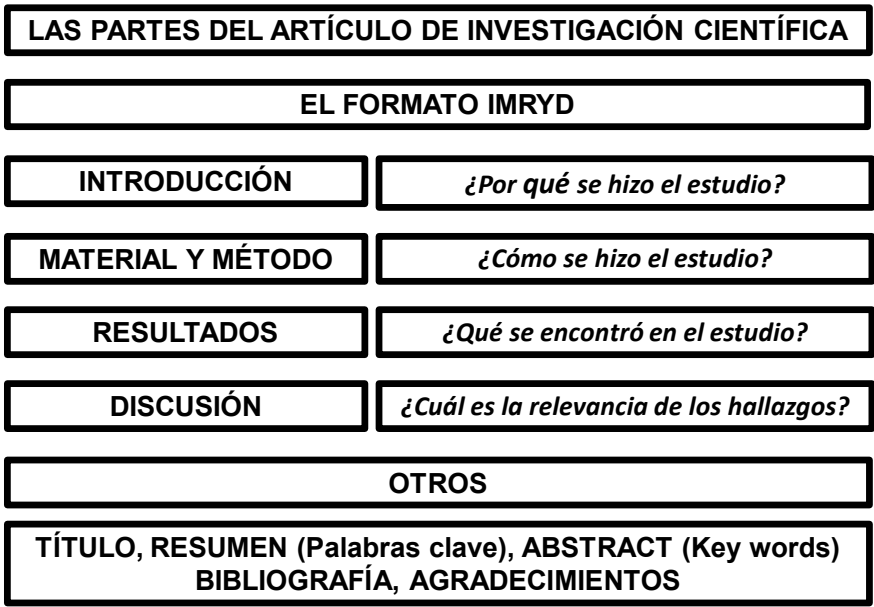

Figura 6. Formato IMRYD de un artículo de investigación científica. 
lo interpretará a la luz de la evidencia generada durante la etapa de contraste empírico. Esta sección suele finalizar con una mención explícita al objetivo del trabajo.

Desarrollo. Se mencionan los procedimientos generales que se siguieron para llevar a cabo la investigación. Su objetivo es exponer y demostrar y consta de tres secciones: (a) Material y Métodos, en la que se describe tanto el material biológico empleado, como los procedimientos seguidos, (b) Resultados, sección eminentemente descriptiva en la que se presentan los datos obtenidos, los que suelen mostrarse en forma de tablas y gráficos para lograr una visualización más comprensible, acompañados de los análisis efectuados y (c) Discusión, sección propiamente especulativa en la que las evidencias generadas en el marco del proceso se enfrentan con la disponible en el cuerpo de conocimientos de la disciplina y se elaboran y formulan explicaciones que permiten extraer algún significado de los datos concretos, para predecir relaciones con otros fenómenos y establecer generalizaciones.

Conclusión. La conclusión de un trabajo científico está íntimamente ligada con la Introducción, ya que debe confirmar o rechazar la hipótesis planteada. También se conecta con la discusión en tanto deriva de ella. Esta conexión da lugar a la conocida circularidad propia del trabajo científico. Posee la característica de síntesis, es decir que debe relacionar las distintas partes de la argumentación y unir las ideas desarrolladas en un marco de coherencia lógica en dos sentidos: hacia el interior del trabajo en relación con la evidencia generada y hacia el exterior del mismo en relación con la red de conocimientos disciplinares en los que se sustenta.
La conclusión es un regreso a la introducción: se cierra sobre el comienzo.

Bibliografía. Esta sección se vincula con dos características de la ciencia a saber, la de ser un juego de discursos referidos en tanto se hace mención a los discursos de otros que nos precedieron y cuya palabra se trae al campo de juego particular de esa instancia investigativa $\mathrm{y}$, a la vez, la de ser un juego de discursos polémicos en el sentido que las ideas del investigador acerca de los hechos deben ser confrontadas con las ideas coincidentes o discrepantes de otros acerca de dichos hechos.

Resumen. Condensa los aspectos esenciales del trabajo. Su finalidad es interesar sobre el contenido del texto, tanto al lector que tiene acceso al trabajo completo como al que accede a los documentos de resúmenes. El resumen, al igual que el título, son promesas de lo que contiene en su interior el artículo que aún no se ha leído, promesas que no deben ser defraudadas.

Las tres secuencias particulares descritas: los momentos del proceso de investigación, los pasos del método hipotético-deductivo y la estructura del artículo de investigación científico guardan entre sí un evidente paralelismo que es puesto claramente en evidencia en la Figura 7.

En este punto resulta necesario reforzar la idea que toda representación esquemática sobre el proceso de investigación corre el riesgo de considerarse un modelo restrictivo, un molde rígido de procedimientos. En realidad la labor científica es un trabajo donde la libertad y la creación cumplen un papel central: no hay, ni puede haber, ninguna receta que garantice un resultado

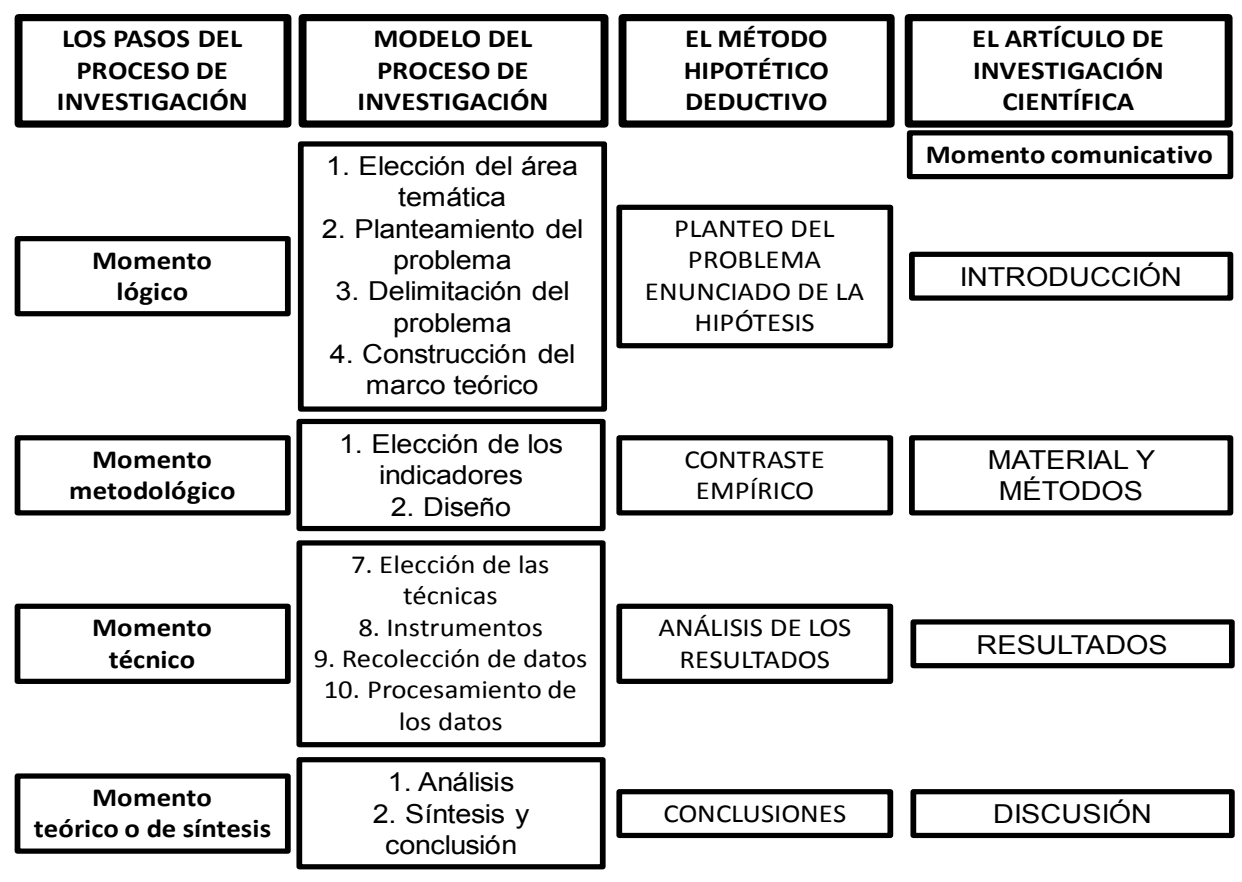

Figura 7. Paralelismo entre las secuencias lógicas que caracterizan al proceso de investigación, al método hipotético-deductivo (como versión del método científico vigente en las ciencias fácticas naturales) y la estructura del artículo de investigación (como instrumento de socialización del producto de la actividad científica). 
positivo para el trabajo del investigador por cuanto las dificultades y los imprevistos son tantos que impiden alcanzar una planificación completa del proceso. La práctica de la investigación implica unir el pensamiento riguroso a la imaginación, la disciplina de trabajo a la inspiración, en dosis variables según las circunstancias. Por eso cualquier esquema tiene por objeto estimular el pensamiento sistemático y la creatividad, siendo una guía para tener en cuenta los principales factores y aspectos que intervienen en el proceso de investigación científica.

\section{A modo de cierre}

Para finalizar este análisis se retoma la analogía planteada en uno de los párrafos iniciales entre el proceso de investigación y un viaje en ómnibus. Es posible ampliar la vinculación entre ambos y pensar el viaje desde el momento en que se toma la decisión de hacerlo, cuando surge la idea, cuando se averiguan detalles y se buscan antecedentes en diversas fuentes, cuando se mantienen conversaciones con otros que hicieron un viaje similar con anterioridad y, después de efectuado el viaje, ya de regreso, se reflexiona sobre todo aquello que quedó por ver en futuros viajes a realizar. Hay por lo tanto, en todo proceso de investigación, un marco histórico que nos informa acerca del pasado del problema y un futuro imaginable vinculado con el contraste de nuevas hipótesis emergentes del mismo proceso (Figura 2). Reconocer tanto ese "antes" y ese "después" como la particularidad que presenta el "durante" que tiene al investigador como protagonista, con similitudes y diferencias del "durante" de otros viajeros que visitaron los mismos lugares, es haber incorporado en gran medida la naturaleza del oficio de investigar en el marco del proceso vinculado con esa aventura que llamamos ciencia.

\section{REFERENCIAS}

1. Asti Vera A. 1968. Metodología de la investigación, Kapelusz, Buenos Aires, $195 \mathrm{p}$.

2. Bunge M. 1988. La ciencia, su método y su filosofía, Siglo XXI, Buenos Aires, 159 p.

3. Bunge M. 2000. La investigación cientifica. Su estrategia y su filosofía, Siglo XXI, México, 805 p.

4. Bunge M. 2002. Diccionario de filosofía, $2^{\circ}$ ed., Siglo XXI, Buenos Aires, $221 \mathrm{p}$.

5. Carpio AP. 2004. Principio de filosofía. Una introducción a su problemática, $2^{\circ}$ ed., Glauco, Buenos Aires, 517 $\mathrm{p}$.

6. Dieterich H. 1999. Nueva guía para la investigación cientifica, Editorial 21, Buenos Aires, 236 p.

7. Estany A. 2006. Introducción a la filosofía de la ciencia, Servicio de Publicaciones, Universidad Autónoma de Barcelona, Barcelona, $266 \mathrm{p}$.

8. Klimovsky G. 1999. Las desventuras del conocimiento cientifico. Una introducción a la epistemología, $4^{\circ} \mathrm{ed} ., \mathrm{AZ}$ Editora, Buenos Aires, 418 p.

9. Pérez Lindo A, Ruiz Moreno L, Varela C, Grosso F, Camós C, Trottini AM, Burke ML, Darín S. 2005. Gestión de conocimiento. Un nuevo enfoque aplicable a las organizaciones y la universidad, Ed. Norma, Buenos Aires, $279 \mathrm{p}$.

10. Rezzónico RC. 2003. Comunicaciones e informes cientificos, académicos y profesionales en la sociedad del conocimiento, Ed. Comunicarte, Córdoba, 227 p.

11. Rubio MJ, Varas J. 1997. El análisis de la realidad en la intervención social. Métodos y técnicas de investigación, Ed. CCS, Madrid, $454 \mathrm{p}$.

12. Sabino CA. 1996. El proceso de investigación, $3^{\circ}$ ed., Lumen-Humanitas, Buenos Aires, $239 \mathrm{p}$.

13. Samaja J. 1999. Epistemología y metodología. Elementos para una teoría de la investigación cientifica, $3^{\circ}$ ed., Eudeba, Buenos Aires, 409 p.

14. Tarrés MC, Montenegro SM, D’Ottavio AE, García Sánchez E. 2008. Lectura crítica del artículo científico como estrategia para el aprendizaje del proceso de investigación. Rev Iberoam Educ 45: 1-8. 